\title{
Should urologists give chemotherapy?
}

\section{Fred Saad, MD, FRCS}

S hould a urologist dedicated to urological oncology give chemotherapy? Should a medical oncologist with little interest in or knowledge of prostate cancer give taxotere? I believe the best answer would be that both work together to optimize patient care, but unfortunately the real world does not always allow the ideal to be possible.

\section{Reality check}

Urologists have been involved in medical therapy of many urological diseases for many decades and have been active players in advancing the nonsurgical therapeutic alternatives in all facets of urology. Patient care in many cancer centres separates therapeutic expertise into 3 groups: 1 group for research, 1 for technical therapeutics (i.e., surgery) and 1 for medical therapeutics (i.e., chemotherapy). This is unfortunate since I am convinced that expertise comes from understanding and being involved in the disease from basic science through to novel therapeutics. I am a believer that it is more important to understand what we are treating than simply what we are treating with. It is clear that in the case of chemotherapy there are prerequisites before we can jump in. There obviously has to be a clear understanding of how the drugs should be given, the precautions, interactions and side effects, and how to handle the complications. This needs to be more than theoretical since things can become problematic if safety nets are not in place. Just as for medical oncologists, with the right environment things can be done safely.

\section{What is needed?}

What is needed is an oncology-focused clinic with dedicated nurses and pharma- cists who are part of the team to ensure quality control, easy access for patients who may be in trouble and easy access to an emergency department with a team experienced in treating patients receiving chemotherapy. One of the most important aspects is to dedicate time to these patients since chemotherapy is only one part of the care of patients with advanced prostate cancer. One also has to treat sufficient numbers of patients to become comfortable in almost all aspects. Do I suggest doing this alone? Absolutely not! There needs to be close interaction with other experts in urology and medical oncology. Obviously, the ideal would be for all involved to work together to optimize care, but when this is not possible or feasible on a continuous basis I do not see why urologists who are willing to take the time and effort to manage cancer patients should not include chemotherapy in their therapeutic armemtariam. I have been actively involved in the medical management of prostate cancer for almost 15 years and have gotten a great deal of satisfaction by working in close collaboration with my medical oncology colleagues and the excellent support staff that make up the oncology team. This team includes nurses, pharmacists and technicians who make it possible for me and other specialists (medical, surgical and gynecological) to administer chemotherapy safely and manage side effects as efficiently as possible. Training, continuing medical education and mentorships are possible to allow those interested in acquiring the basis for administering chemotherapy, just as we obtain training in other areas that were not covered during residency or fellowships. At the present time, training in urological oncology in many countries is being reviewed since it is obvious that excellence
The purpose of the Point / Counterpoint section is to encourage vigorous and informed discussion on controversial issues in urology through the presentation of diverse opinions. We aim for a dispassionate discussion of controversies, recognizing that strong passions may exist in support of some positions. 
in urological oncology needs to go beyond research and the operating room.

\section{Conclusion}

My experience in chemotherapy, although timeand energy-consuming, has given me a great deal of satisfaction, and patients appear to appreciate my involvement in their care until the end. It has also allowed me to be an active participant in the progress we've made in the management of hormone refractory prostate cancer in daily practice and through clinical trials. I believe this would have been difficult if I were on the outside looking in. I am also convinced that if urology, as a specialty, is going to maintain a leadership role in the field of research in urological oncology, we will have to remain intimately involved in medical therapeutics whether or not we actually take the responsibility of prescribing chemotherapy.
With the progress in medicine evolving rapidly and the limited role that surgery will play in oncology of the future, it will be essential for us as urologists to be proactive rather than passive in the field of urological oncology. History has shown that urologists are able to and more than capable of taking on and embracing new challenges in our ever-changing specialty. I think this is at least one of the reasons why urology is so enjoyable.

Professor of Surgery and Urology, University of Montreal, Director of Urologic Oncology Centre Hospitalier de I'Université de Montréal, Montréal, Que.

The positions provided in the Point/Counterpoint series are presented as general information and do not necessarily reflect the personal opinions of the authors.

This article has been peer reviewed.

Competing interests: None declared.

Correspondence: Dr. Fred Saad, Université de Montréal Directeur, Urologie-Oncologie, CHUM, 1560 Sherbrooke E., Montréal QC H2L 4MI; FredSaad@videotron.ca

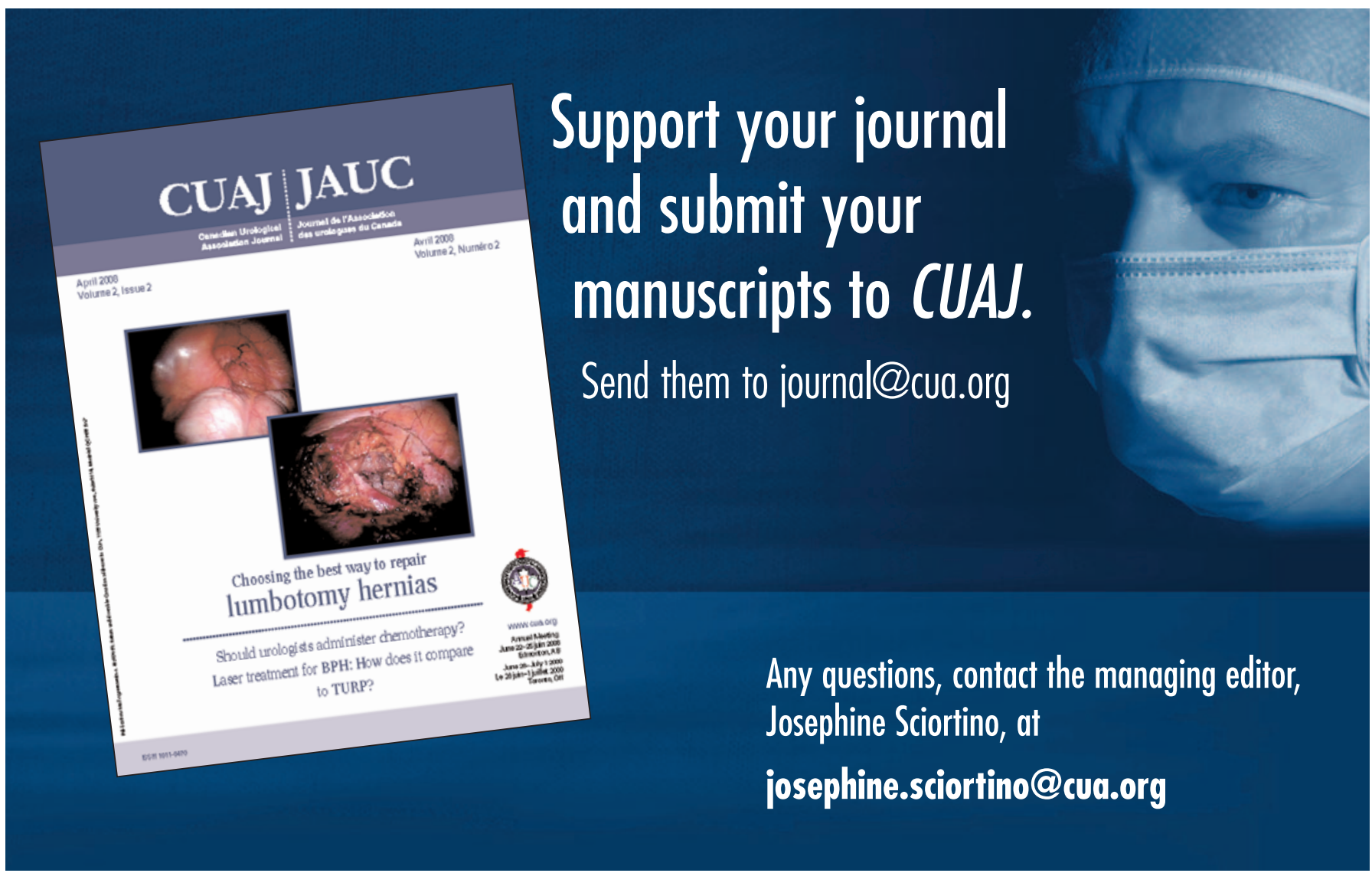

